\title{
BENEFICIOS DEL CUMPLIMIENTO Y GESTIÓN DE RIESGOS
}

\section{ARTÍ́CULO ORIGINAL}

SILVA, Lilian Reis da ${ }^{1}$

SILVA, Lilian Reis da. Beneficios del cumplimiento y gestión de riesgos. Revista Científica Multidisciplinar Núcleo do Conhecimento. Año. 06, Ed. 12, Vol. 04, págs. 123-147. Diciembre 2021. ISSN: 2448-0959, Enlace de acceso: https://www.nucleodoconhecimento.com.br/administracion-de-empresas/beneficiosdel-cumplimiento, DOI: 10.32749/nucleodoconhecimento.com.br/administracion-deempresas/beneficios-del-cumplimiento

\section{RESUMEN}

El cumplimiento es un programa que tiene como objetivo proteger a las organizaciones de la ocurrencia de fraude financiero, corrupción, comportamiento inapropiado y/o conducta de los empleados vinculados a ellas, evitando que su buena reputación y solidez financiera se vea socavada. Es una herramienta cuyo propósito es establecer reglas, estándares y lineamientos para los procesos internos dentro de las organizaciones. Fue desarrollado en los Estados Unidos en 1970, y sus prácticas se transformaron en un instituto legal, a través de la promulgación de la Ley Pionera FCPA (Foreign Corrupt Practices Act), motivada por el caso Watergate, que involucró al entonces presidente Richard Nixon y miembros de su gobierno, mediante el pago de sobornos en investigaciones a favor de su reelección. En este contexto, este artículo tiene como pregunta orientadora: ¿cómo colaboran los beneficios que ofrece el programa de Cumplimiento y Gestión de Riesgos para reducir los riesgos de fraude, actos ilícitos y corrupción dentro de las organizaciones? El objetivo de este estudio fue presentar los beneficios que brinda la adopción del programa de Cumplimiento y Gestión de Riesgos en empresas públicas y privadas, en sus procesos internos y relaciones con su segmento de actividad, y cómo colaboran para mitigar el fraude. Se

\footnotetext{
${ }^{1}$ Postgrado en Dirección de Empresas, economista y técnica contable.
}

RC: 103916

Disponible: https://www.nucleodoconhecimento.com.br/administracion-deempresas/beneficios-del-cumplimiento 
adoptó como metodología la investigación bibliográfica, discutiendo los beneficios que generan los programas de prevención del fraude. Se descubrió que los programas de cumplimiento y gestión de riesgos brindan beneficios efectivos en la protección de riesgos, mitigando el fraude y la corrupción, junto con nuevas soluciones de gobernanza de Tecnología de la Información (TI), como la Enterprise Governance, Risk Management and Compliance (EGRC).

Palabras clave: Fraude dentro de las organizaciones, Prevención de riesgos de corrupción, Cumplimiento, Compliance.

\section{INTRODUCCIÓN}

Discutir los Beneficios del Compliance y la Gestión de Riesgos implica presentar conceptos sobre qué es un programa de cumplimiento y cuáles son los riesgos que corren las empresas cuando no adoptan este tipo de programas, en vista de los diferentes tipos de fraude, incluyendo corrupción, lavado de dinero, manipulaciones con información interna y/o cuando los empleados cometen acciones que pueden dañar la imagen de una organización, incluso puede causar pérdidas financieras.

Según Michelotti (2020, p. 6), la adopción de un programa de Compliance y gestión de riesgos brinda transparencia a las organizaciones, demostrando la existencia de una conducta ética; además, colabora con las prácticas de buen Gobierno Corporativo, que es el "conjunto de procesos por los que se gestionan las empresas".

Según Sobreira Filho, Leite e Martins (2019, p. 23), "el programa de compliance es una herramienta corporativa cuya metodología para su implementación se basa en la ética empresarial y la integridad como su mayor valor".

Se define el término cumplimiento (también llamado compliance), nacido del verbo inglés, "to comply", resumido por Coelho y Santos Jr (2021, p. 8) como "satisfacer las imposiciones de orden jurídico $u$ orden interno de la empresa" (grifos del original). A este concepto, Gonsales (2016) agrega que "si en un principio, el 
cumplimiento significaba "seguir las reglas", de las grandes investigaciones brasileñas, también llegó a significar "seguir la ley anticorrupción".

Cabe destacar que el cumplimiento se aplica a empresas públicas o privadas, de diferentes tamaños y segmentos, ubicadas en todos los países, organizaciones que tienen el anhelo de actuar con ética, moral y en la promoción del desarrollo humano, social, económico y financiero.

Este artículo fue desarrollado como una investigación bibliográfica, para lo cual se definió la siguiente pregunta: ¿cómo colaboran los beneficios que ofrece el programa de Cumplimiento y Gestión de Riesgos para reducir los riesgos de fraude, ilícito y corrupción dentro de las organizaciones? A su vez, el objetivo general fue presentar los beneficios que brinda la adopción del programa de Cumplimiento y Gestión de Riesgos en empresas públicas y privadas, en sus procesos internos y relaciones con su segmento de actividad, y cómo colaboran para la mitigación del fraude.

\section{CUMPLIMIENTO}

\subsection{CONCEPTO}

Para que una empresa sea considerada confiable, inspirando seguridad en su segmento de actividad y ante la sociedad, es importante que tenga valores basados en la moral, la ética y la justicia. Debe actuar con transparencia, lo que le permite construir su credibilidad, y también atraer talentos humanos que mejoren su capacidad de acción, apuntando a su crecimiento (JORGE y TOMAZ, 2018).

En esta perspectiva, para que una organización actúe con ética, buenos principios y competitividad, la lucha contra el fraude es un tema fundamental, inhibiendo cualquier tipo de ilícito, a través de la adopción de prácticas y procesos para proteger sus valores y objetivos, adoptando un programa de cumplimiento.

El cumplimiento presupone "el cumplimiento y el respeto de las normas y reglamentos". Según Antonik apud Jorge y Tomaz (2018, p. 108), cumplimiento

RC: 103916

Disponible: https://www.nucleodoconhecimento.com.br/administracion-deempresas/beneficios-del-cumplimiento 
(también llamado compliance) viene del inglés, " cumplir ", que traducido significa "reglas, instrucciones, normas, directrices o simplemente responder a una orden".

Coelho y Santos Júnior (2021, p. 8) señalan que "el objetivo de las normas de cumplimiento es centrarse en el resultado a alcanzar, es decir, evitar los riesgos derivados de la comisión de conductas personales u organizativas consideradas ilegales o inconsistentes con los principios, misiones, visión u objetivos de una empresa".

Con el tiempo, entre 1907 y 1977, han surgido leyes aplicables en los Estados Unidos con alcance internacional también, para controlar y castigar a los ciudadanos, empresas y agencias gubernamentales que participan en acciones de corrupción.

Según Nascimento (2018), cumplimiento fue creado en los Estados Unidos, alrededor de la década de 1970, elevado como un instituto legal a partir de la promulgación de la Ley Pionera para Combatir la Corrupción o - Foreign Corrupt Practices Act (FCPA). Dicha legislación se desarrolló e implementó después de que ocurriera el caso Watergate, que culminó con la renuncia del entonces presidente de los Estados Unidos, Richard Nixon.

Furtado y Rocha (2015, apud MICHELOTTI, 2020, p. 7-8) informan que en el período previo a la Ley FCPA, las prácticas del capitalismo en la apertura de nuevos mercados incluían el pago de sobornos, "considerado un paso necesario y natural en el entorno corporativo. Existía la posibilidad más clara de que el soborno se contabilizara como gastos en el balance. $Y$ el soborno para obtener contratos o concesiones en estos países era parte de las reglas del juego".

Dado que la corrupción es un problema global, e involucra -desde el principio- a gobiernos, personas, instituciones públicas y/o privadas, Ubirajara Costódio Filho, a la que se refiere Nascimento (2021) afirma que es algo que perjudica a todas las esferas sociales, con respecto al medio ambiente, la libre competencia, las finanzas públicas, el comercio internacional, democracia y preceptos republicanos. 
Todavía sobre la corrupción, Nascimento (2021, s.p.) también informa Eduardo Cambi, para destacar que:

\begin{abstract}
a corrupção atrasa o desenvolvimento econômico e social. Restringe a vontade soberana do povo. Apropria a coisa pública para a realização de interesses privados. Gera promiscuidade entre o poder público e o poder econômico. Concentra renda. Ressalta privilégios e desigualdades. Impede a universalização de políticas públicas e a concretização de direitos fundamentais. Destrói a cidadania e enfraquece a democracia.
\end{abstract}

El propósito de la Ley Foreign Corrupt Practices Act (FCPA), ou Ley Americana contra las Prácticas Corruptas, era castigar las acciones fraudulentas de las empresas que ofrecían/pagarían sobornos al servicio público, con el objetivo de expandir las empresas en todo el mundo. A partir de la adopción de las prácticas prescritas en la ley FCPA, todas las organizaciones estaban obligadas a seguir regulaciones internas y externas basadas en las cuestiones éticas y morales necesarias para las relaciones comerciales y económicas con sus socios (NASCIMENTO, 2018).

\title{
2.2 APARICIÓN DE LEGISLACIÓN PARA PRÁCTICAS DE CUMPLIMIENTO
}

Según Nascimento (2018, p. 26), el movimiento global para combatir el soborno y la práctica arraigada de la corrupción" comenzó con la Ley Foreign Corrupt Practices Act (FCPA), o Ley Americana contra las Prácticas Corruptas. Este autor aclara que el "grado de corrupción en un país determinado varía según la forma en que las multinacionales actúan allí".

Entre las tasas de corrupción verificadas, Nascimento (2018, p. 26-27) reporta la proporción de empresas en todo el mundo donde persiste la corrupción ilícita y el lavado de dinero:

$23 \%$ das empresas na União Europeia e Europa Ocidental; $66 \%$ das empresas na América Latina; $64 \%$ das empresas na Ásia; 95\% das empresas na Europa Ocidental e da Ásia Central; $84 \%$ das empresas do Oriente Médio e do Norte da África; $90 \%$ das empresas da África Subsaariana.

RC: 103916

Disponible: https://www.nucleodoconhecimento.com.br/administracion-deempresas/beneficios-del-cumplimiento 
Este autor (NASCIMENTO, 2018, p. 26-27) dice que la globalización económica "trajo consigo el crimen global de corrupción relacionado con el lavado de dinero y la corrupción offshore".

Se observa, por lo tanto, que la literatura sobre programas de cumplimiento y gestión de riesgos muestra que, en un momento dado, varios países y sus gobiernos decidieron enfrentar prácticas ilegales -tanto en instituciones públicas como en organizaciones privadas- que desde entonces, se han visto obligados a adoptar medidas punitivas y restrictivas para los ciudadanos y países que cometen fraude, corrupción y lavado de dinero, entre otros, dando lugar a tratados internacionales.

Los países que adoptan programas de prevención del fraude son signatarios de la Organización para la Cooperación y el Desarrollo Económicos (OCDE), entre ellos: Alemania, Australia, Austria, Bélgica, Canadá, Chile, Corea, Dinamarca, Eslovenia, España, Estados Unidos, Estonia, Finlandia, Francia, Grecia, Hungría, Irlanda, Islandia, Israel, Italia, Japón, Letonia, Luxemburgo, México, Noruega, Nueva Zelanda, Países Bajos, Perú, Polonia, Portugal y Reino Unido (NASCIMENTO, 2021, s.p.).

Para aclarar cómo estos países optaron por tomar la adopción de buenas prácticas de gobierno corporativo, es importante lograr el surgimiento de instituciones y leyes estadounidenses que permitieron la adopción de institutos de protección con respecto a los diferentes tipos de ilícitos contra las organizaciones, causando la promulgación de leyes para los mismos fines en todo el mundo.

En 1907, se celebró la Conferencia de La Haya, que es "una corte internacional de justicia para investigar conflictos internacionales". Con él, se desarrolló un alcance para la fundación del Banco de Pagos Internacionales (BPI) (Bank for International Settlements - BIS), con el objetivo de promover "la cooperación entre los bancos centrales para lograr una mayor estabilidad financiera". Este objetivo es considerado por los expertos como "las primeras raíces del cumplimiento "(NASCIMENTO, 2021, s.p.).

RC: 103916

Disponible: https://www.nucleodoconhecimento.com.br/administracion-deempresas/beneficios-del-cumplimiento 
En abril de 1948, la Organización Europea para la Cooperación Económica (OECE) nació en Portugal, con el objetivo de la cooperación económica entre los países europeos. Su composición inicial contaba con 18 países, a destacar: Austria, Bélgica, Dinamarca, Francia, Grecia, Holanda, Irlanda, Islandia, Italia, Luxemburgo, Noruega, Portugal, Reino Unido, Suecia, Suiza, Turquía y Alemania Occidental.

También en 1948 fue la Organización de los Estados Americanos (OEA) (Organization of American States - OAS), basada en cuatro pilares: "democracia, derechos humanos, seguridad y desarrollo". (NASCIMENTO, 2021, s.p.).

Aún así Nascimento (2021, s.p.), destaca lo que Sanches y Renee (2017, p. 18) defienden sobre la OEA: "esta convención tiene como objetivo promover y fortalecer los mecanismos necesarios para ayudar a prevenir, detectar y sancionar la corrupción en el ejercicio de las funciones públicas, así como los actos de corrupción específicamente vinculados a su ejercicio".

En diciembre de 1960, los miembros de la OECE firmaron con los Estados Unidos y Canadá una nueva Convención que creó la Organización para la Cooperación y el Desarrollo Económicos (OCDE), y luego reemplazó a la OECE. Así, en 1961 surgió la Organización para la Cooperación y el Desarrollo Económicos (OCDE) (Organisation for Economic Co-operation and Development (OECD), en sucesión de la Organización para la Cooperación Económica Europea, cuyos objetivos eran "abarcar el desarrollo económico y la estabilidad financiera de los países miembros" (NASCIMENTO, 2021, s.p.).

En 1975, se fundó el Comité de Supervisión Bancaria de Basilea (BCBS) (Basel Committee on Banking Supervision - BCBS), cuyo propósito era "regular y supervisar las mejores prácticas financieras". En 1998, promovió una uniformidad de las normas aplicables a las instituciones financieras, con el objetivo de mantener las buenas prácticas de Gobierno Corporativo (NASCIMENTO, 2021, s.p.).

En 1977, ante el escándalo de Watergate, se desarrolló la Ley Americana Contra las Prácticas de Corrupción en el Extranjero (FCPA), siendo la 1ra. ley anticorrupción, RC: 103916

Disponible: https://www.nucleodoconhecimento.com.br/administracion-deempresas/beneficios-del-cumplimiento 
emitida posteriormente en 1988 y 1998. Según Nascimento (2021, s.p.), con el propósito de 'adoptar sanciones civiles, penales y administrativas a personas físicas y jurídicas contra las prácticas corruptas, la Foreign Corrupt Practices Act (FCPA), o la Ley Americana contra las Prácticas Corruptas (NASCIMENTO, 2021, s.p.).

Cabe destacar que el FCPA califica como "delito el pago de sobornos directa o indirectamente, pagos a funcionarios públicos extranjeros, partidos políticos, el pago no registrado de manera concisa, debe haber libros de registros contables" (NASCIMENTO, 2021, s.p.).

Dichas investigaciones fueron abordadas por la Fundación Getúlio Vargas (FGV) (2021, s.p.), informando del surgimiento de la Securities and Exchange Commission(SEC), que es "una organización administrativa para supervisar y combatir las prácticas de corrupción en las transacciones internacionales".

La SEC "encontró prácticas de pagos ilegales a funcionarios públicos por parte de un promedio de 400 empresas privadas y públicas estadounidenses, y extranjeras también". En tal declaración, la SEC encontró que "juntos pagaron más de USD 300 millones en sobornos a funcionarios de gobiernos extranjeros" (FGV, 2021, s.p.).

En 1989, para una acción conjunta con la OCDE, se creó el Grupo de Acción Financiera Internacional (GAFI) (Financial Action Task Force - FATF), destinado a la formulación de políticas y las reformas legislativas y reglamentarias, basadas en las leyes internacionales, destinadas a combatir el lavado de dinero y el financiamiento del terrorismo. El GAFI también actúa en la composición de los mecanismos de evaluación periódica de otros países (NASCIMENTO, 2021, s.p.).

En la práctica, el texto de la OEA trajo la Convención Interamericana contra la Corrupción, firmada en 1996, destinada a "proteger los intereses de los Estados Unidos en términos de terrorismo y corrupción".

En 2002, se implementó la Ley Sarbanes Oxley (Sarbanes-Oxley Act - SARBOX) para promover mejor "la gobernanza corporativa, el monitoreo y la prevención de prácticas

RC: 103916

Disponible: https://www.nucleodoconhecimento.com.br/administracion-deempresas/beneficios-del-cumplimiento 
nocivas, aumentando los controles y la transparencia". Con la creación del Cuerpo de Supervisión del Trabajo de Auditores Independientes, también instituyó auditorías independientes para casos sospechosos, reforzando "la responsabilidad por fraude corporativo o criminal, aumentando el nivel de divulgación de información financiera, aumentando las penas por delitos de cuello blanco (NASCIMENTO, 2021, s.p.).

En 2010, surgió en el Reino Unido la Ley de Soborno del (UK Bribery Act (BA), que tipifica cuatro delitos: "corrupción activa de individuos públicos y privados, corrupción pasiva de individuos públicos y privados, corrupción de funcionarios públicos extranjeros, fracaso de las empresas para prevenir la corrupción" (NASCIMENTO, 2021, s.p.).

A su vez, Madruga y Belloto desarrollaron una guía que presenta 10 pilares que, una vez adoptados, permiten la implementación de un Programa de Cumplimiento eficiente (NASCIMENTO, 2018, p. 8-9):

1. Compromisso de todos os níveis da gerência e política anticorrupção claramente articulada; 2. Código de conduta e política e procedimentos de compliance; 3. Fiscalização interna do programa, autonomia da área de compliance e recursos adequados; 4. Avaliação dos riscos da empresa; 5. Treinamentos e aconselhamento contínuo; 6 . Incentivos e medidas disciplinares; 7. Investigações (due diligence) de terceiros e de pagamentos; 8. Sistema interno de denúncias (possibilitando denúncias confidenciais) e sistema de investigações internas; 9. Melhoria contínua: testes e revisões periódicas do programa de compliance; 10. Fusões e Aquisições: Investigações (due diligence) pré-fusões e aquisições e integrações pós-aquisições e fusões.

Con respecto al Brasil, y a pesar de la promulgación de la Ley № 12.846/13, cuyo propósito es la adopción de medidas legales y procedimientos internos que deben seguir las organizaciones mediante la adopción de un código de conducta para combatir la corrupción, el ingreso a la OCDE aún no ha sido oficializado por este órgano, en vista de los constantes casos de corrupción, fraude y lavado de dinero que surgen aquí (DURÃES y RIBEIRO, 2020).

RC: 103916

Disponible: https://www.nucleodoconhecimento.com.br/administracion-deempresas/beneficios-del-cumplimiento 


\subsection{LAS PRÁCTICAS FRAUDULENTAS $Y$ LAS PÉRDIDAS CAUSADAS POR TERCEROS A LAS ORGANIZACIONES}

Cuando las personas vinculadas a una organización determinada adoptan comportamientos que dañan la imagen de la empresa, sus acciones erróneas causan repercusiones negativas en la misma, así como en toda la sociedad. Hay muchos casos de conductas reprobables que sacuden no solo la imagen, sino que también traen pérdidas, multas y a veces quiebras.

Con respecto a los diferentes tipos de fraude dentro de las organizaciones e instituciones gubernamentales, como la filtración de información confidencial, la corrupción pasiva/activa, el lavado de dinero, entre otros, Gonsales (2016) afirma que todos los estudios relacionados con dicho fraude argumentan que en todas las empresas ocurren. Por lo tanto, cuanto más crea su líder que no está sucediendo nada malo, más vulnerable se vuelve su organización.

En Brasil, existen prácticas fraudulentas conocidas en toda la sociedad, como "falsificar la licencia de un estudiante o robar líneas de televisión por cable", a las que se refieren Condé et al. (2015, p. 95); son actos que consisten en demostrar la mala fe de unos y su clara intención de engañar a otros. Desafortunadamente, son acciones practicadas por muchas personas, pero que con el tiempo se han vuelto tan comunes que terminan siendo aceptadas en la cultura brasileña. Son actos que demuestran una mentalidad y un comportamiento poco éticos e ilegales; sin embargo, se consideran no perjudiciales para la sociedad, lo cual es una falsedad.

En comparación con las acciones anteriores, Condé et al. (2015, p. 95) sugiere que si las prácticas poco éticas, fraudulentas y corruptas fueran llevadas a cabo por ejecutivos de alto rango de una organización, serían consideradas dañinas e inaceptables por la sociedad.

En este sentido, Cossenzo (2015, p. 35) informa Oliveira (2012, p. 115) para definir que "un fraude financiero se lleva a cabo a partir de actos voluntarios de su agente,

RC: 103916

Disponible: https://www.nucleodoconhecimento.com.br/administracion-deempresas/beneficios-del-cumplimiento 
con la clara intención de perjudicar financieramente a otros". Este autor continúa explicando que el fraude puede ser (i) interno, si es cometido por empleados contra la organización; (ii) externo - si es cometido por terceros que no son miembros de la empresa; iii) mixto: si las acciones fraudulentas son llevadas a cabo por agentes internos y externos.

Según esta explicación, cabe destacar un concepto relacionado con el fraude financiero, considerado como "algo inherente al negocio" de este tipo de instituciones, clasificándose como riesgos operacionales relacionados tanto con este segmento como con "las personas, los procesos y la tecnología" (COSSENZO, 2015, p. 35).

Debe quedar claro que el cumplimiento abarca todo tipo de fraude que pueda ocurrir en diferentes segmentos de negocio, es decir, el cumplimiento busca mitigar los riesgos que puedan ser causados por conductas personales $u$ organizacionales (independientemente de si se encuentran en el segmento financiero o no), si no actúan de acuerdo con la conducta ética y de acuerdo con la política de la empresa.

\subsection{SUCESOS PERJUDICIALES PARA LA REPUTACIÓN DE LAS EMPRESAS ESTADOUNIDENSES}

Según Haussen (2021, p. 9), "la reputación de una organización puede verse fuertemente sacudida por un comportamiento desconectado derivado de la falta de implementación de un Programa de Cumplimiento",como lo demuestran los casos de corrupción que han ocurrido en los Estados Unidos, que han sontudecido en los medios de comunicación a lo largo de los años, incluido el Caso Watergate, que condujo a la promulgación de la Ley FCPA, además de otros mencionados a lo largo de este artículo.

Entre los numerosos casos de acciones lesivas a la imagen de las grandes empresas estadounidenses, comprometiendo su credibilidad, se destacarán aquí algunos que llamaron mucho la atención en los medios de comunicación, como lo demuestran algunos informes ubicados en medios electrónicos. Se trata de historias que relatan

RC: 103916

Disponible: https://www.nucleodoconhecimento.com.br/administracion-deempresas/beneficios-del-cumplimiento 
cómo la falta de implementación de un programa de cumplimiento para guiar procesos y personas dentro de una organización termina permitiendo que algunos empleados empañen la imagen y reputación de empresas conocidas a nivel mundial, con acciones reprobables.

El primer informe de Vaz (2012) publicado por exame Magazine trae un caso que ocurrió en el gigante Apple, cuyo texto completo se presenta aquí.

A Empresa Apple foi acusada pelo Senado nos Estados Unidos de usar filiais no exterior para evitar o pagamento de bilhões de dólares em impostos. "A Apple não apenas transferiu seus lucros para um paraíso fiscal estrangeiro, mas criou um 'Santo Graal' de evasão fiscal ao montar entidades no exterior para driblar o fisco", disse o senador democrata Carl Levin.

Uma reportagem do New York Times revelou como a Apple tem feito para driblar bilhões de dólares em impostos nas suas operações nos Estados e em outros países. Segundo Martin A. Sullivan, um ex-economista do Tesouro americano, sem as táticas utilizadas para a gestão dos impostos, a empresa liderada por Tim Cook teria deixado pelo menos mais 2,4 bilhões de dólares em taxas federais nos EUA no ano passado. A empresa declarou ter pagado 3,3 bilhões de dólares em impostos ao redor do mundo em 2011 sobre os lucros de 34,2 bilhões de dólares, uma taxa de $9,8 \%$. A companhia declara que $30 \%$ dos lucros vem dos Estados, caso o percentual pulasse para $50 \%$, os pagamentos saltariam em 2,4 bilhões de dólares. "É razoável esperar que 70\% dos lucros viriam dos Estados Unidos", calcula.

RC: 103916

Disponible: https://www.nucleodoconhecimento.com.br/administracion-deempresas/beneficios-del-cumplimiento 
Figura 1 - Símbolo de Apple

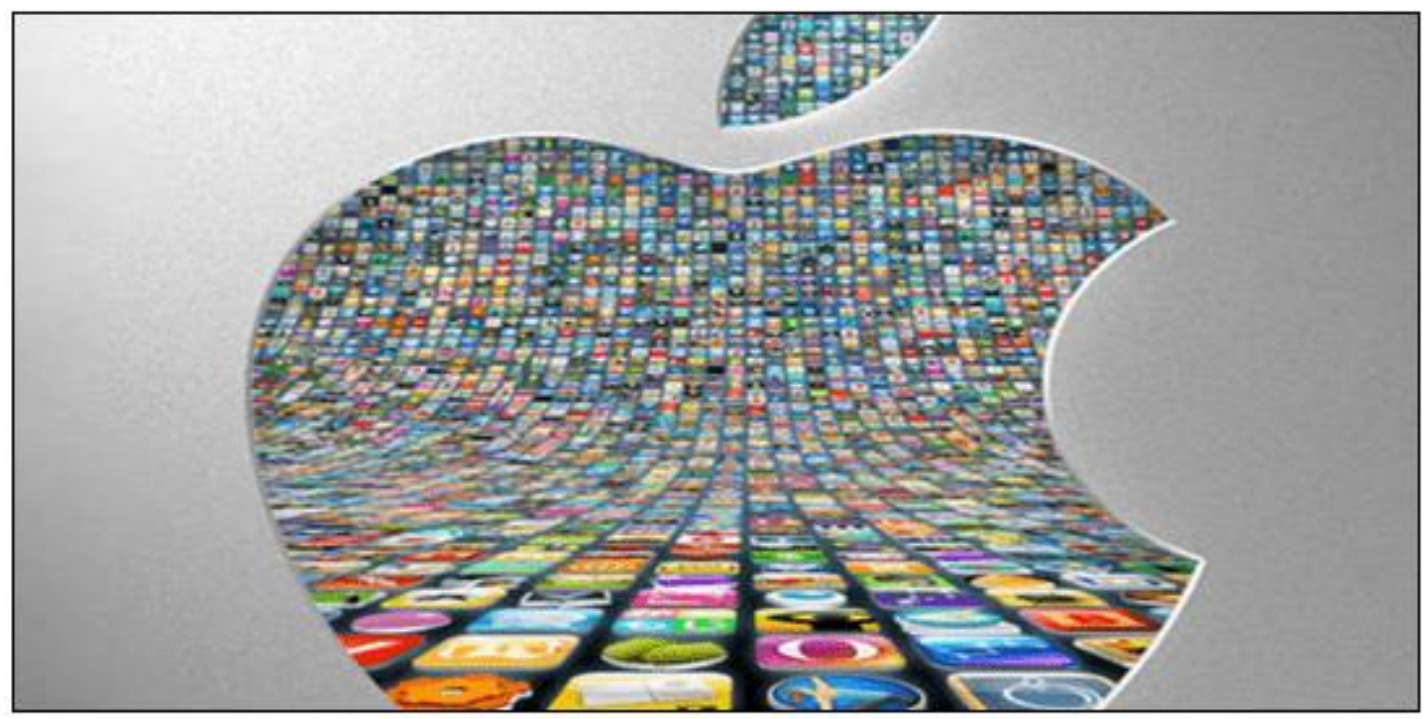

Fuente: Vaz (2012)

Con respecto a las posibles causas que pueden comprometer la credibilidad de las organizaciones, Souza; Maciel-Lima y Lupi $(2018$, p. 13) se refieren a Gonsales (2016), para explicar que:

o valor de uma empresa está ligado à sua reputação e no caso de uma empresa ser citada em jornais e meios de comunicação, ainda que seja um ato ilícito isolado, acometido por um funcionário, a empresa sofrerá abalo a reputação e passa a ser conhecida no mercado como uma empresa corrupta.

El segundo informe se refiere a dos situaciones diferentes, que ocurrieron en dos unidades diferentes en restaurantes norteamericanos de la Red Taco Bell, fast food inspirada en la cocina mexicana, que sigue el modelo de McDonalds.

El primero está en el informe de melo (2014) publicado por Revista Exame, cuyo texto sigue a continuación.

Publicar uma foto de si mesmo fazendo xixi em um prato do Taco Bell, nos Estados Unidos, não custou ao atendente Cameron Jankowski apenas o emprego. Indignados, hackers divulgaram os dados pessoais do "brincalhão" na internet, segundo o Huffington Post. Em 2012, Jankowski postou imagem no Twitter, mas disse que não chegou a servir a comida no restaurante. Ele chegou a deletar sua conta, mas acabou sem seu emprego na rede.

RC: 103916

Disponible: https://www.nucleodoconhecimento.com.br/administracion-deempresas/beneficios-del-cumplimiento 
Figura 2 - Panel electrónico Taco Bell

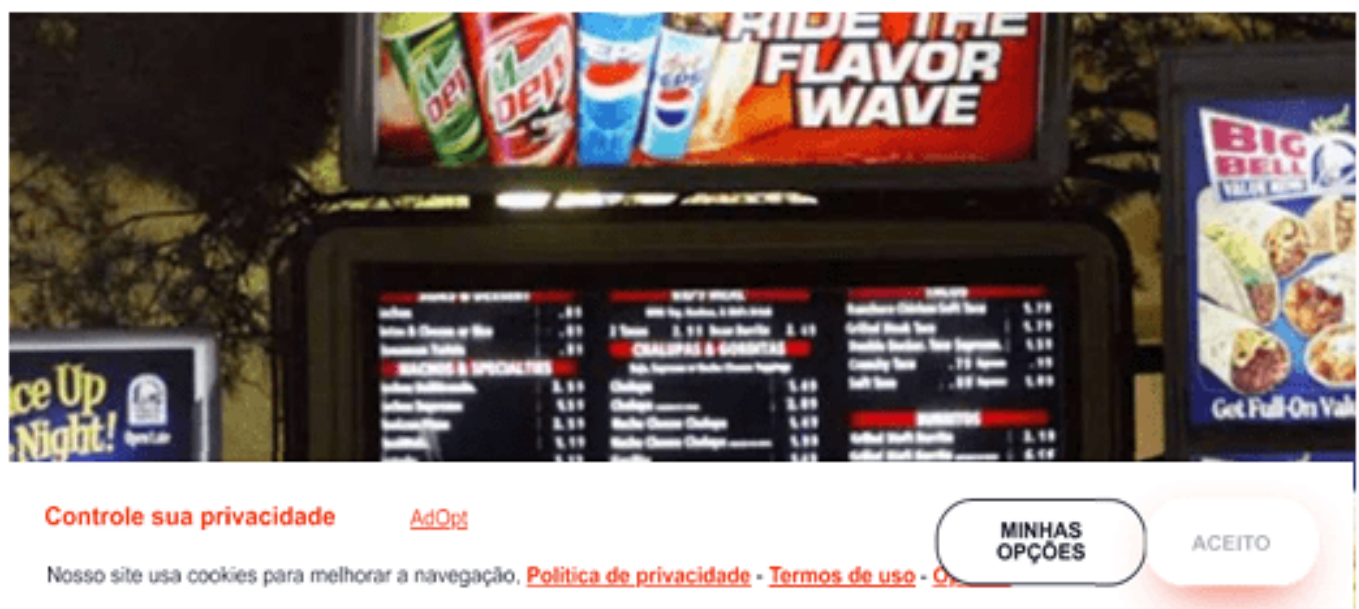

Fuente: Melo (2014)

En 2013, la misma red habría pasado por otro momento que causó daño a su credibilidad. El informe es de Revista Veja On Line (2014), cuyo texto sigue en su totalidad.

Uma imagem de um funcionário da rede de comida mexicana Taco Bell lambendo uma pilha de tortilhas foi postada na página da empresa no Facebook. A foto, de autoria do usuário Jj O'Brien Nolan, é seguida da legenda "isto com certeza diz muito sobre seus empregados, o tratamento da comida e o que eles postam na internet.

Apesar de inicialmente acreditar que a foto fosse uma brincadeira, a empresa demitiu o empregado. Além disso, em resposta, a Taco Bell divulgou um comunicado oficial, além de comentar a postagem de Nolan no Facebook. A empresa se defendeu afirmando que todas as unidades têm rígidos procedimentos de manuseamento e tolerância zero para violação dos alimentos.

RC: 103916

Disponible: https://www.nucleodoconhecimento.com.br/administracion-deempresas/beneficios-del-cumplimiento 
Figura 3 - Empleado de Taco Bell lamiendo un montón de tortillas

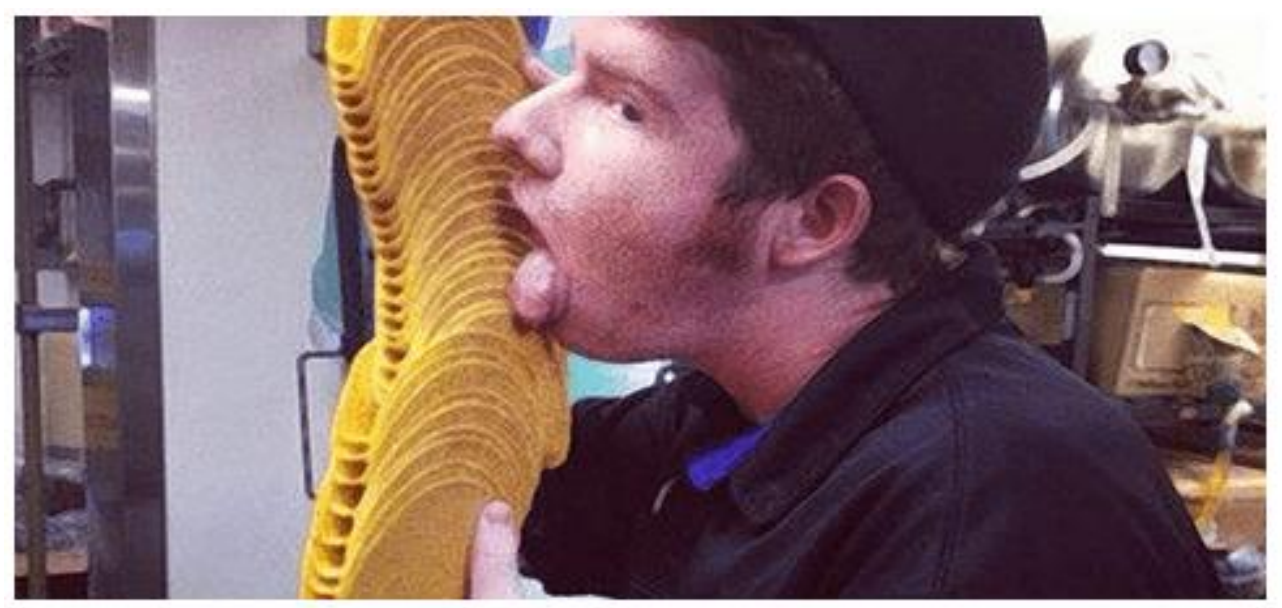

Fuente: Veja en línea (2014)

Un tercer caso a mencionar fue publicado en un reportaje de Melo (2014, publicado por Revista Exame, que ocurrió en uno de los restaurantes de la Red KFC, norteamericano, cuyo texto original se reproduce aquí.

Este fue el caso en el que una ex empleada de la cadena de restaurantes KFC en los Estados Unidos publicó una foto en Facebook cuando literalmente estaba lamiendo el puré de papas. Ella y su amigo fotógrafo fueron despedidos. La red tuvo que justificarse diciendo que la comida no estaba servida. 
Figura 4 - Empleado de KFC lamiendo puré de papas
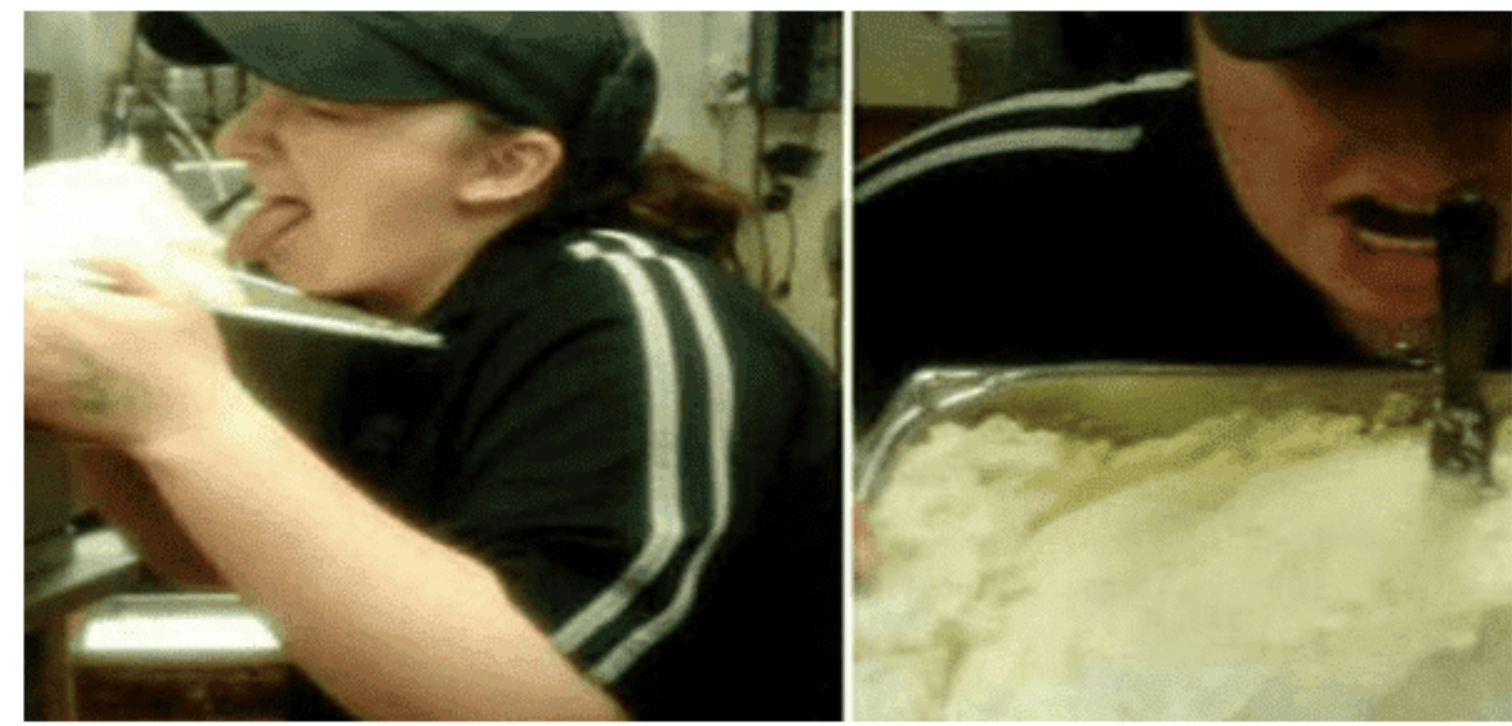

Fuente: Melo (2014)

Los casos de conducta poco ética presentados reflejan la importancia en la adopción de prácticas de Cumplimiento y Gestión de Riesgos. Desde la perspectiva de Coelho y Santos Júnior (2021, p. 26), "no basta con que la empresa tenga una condición financiera para invertir en publicidad y marketing para liderar el mercado".

Lo que sí se verifica, en la práctica, es que las organizaciones deben mantenerse al día permanentemente, atentas a las nuevas prácticas de marketing, además de adoptar estrategias que les permitan ser competitivas en su segmento de actividad, ofreciendo mejores servicios, precios competitivos y dando opciones de elección a sus consumidores objetivo.

En este sentido, Coelho y Santos Júnior (2021, p. 27) argumentan que "entre los factores que influyen en el nivel de competitividad empresarial se encuentran: capacidad de innovación, productos y servicios de calidad, diferenciales comerciales, capacidad de producción y entrega, alcance geográfico, acciones de marketing; entre otros". 


\section{RIESGOS DE CUMPLIMIENTO}

Al no adoptar programas de prevención y mitigación del fraude en sus procesos organizativos, las empresas están sujetas al evento de diferentes eventos ilícitos, ya que "el fraude ocurre en todas las empresas". Además, son vulnerables a dos tipos de riesgos muy dañinos: la sacudida de su reputación y los castigos previstos en la nueva ley anticorrupción (GONSALES, 2016).

El término "Riesgo de Cumplimiento" se refiere a los riesgos a los que están sometidas las empresas por sanciones regulatorias, pérdidas financieras y/o pérdidas causadas a su imagen, riesgos derivados del incumplimiento de la normativa, códigos de conducta y buen comportamiento (COELHO y SANTOS JR, 2021, p. 8).

Según Gonsales (2016) el 43\% de las empresas brasileñas aún no han adoptado programas y políticas de Cumplimiento para sus procesos y protección, pero que pronto todos se verán obligados a hacerlo, con el objetivo de proteger su reputación, ya que "el valor de una empresa está relacionado con su reputación.

Es una realidad que ha transformado el entorno comercial e industrial nacional, surgida de las investigaciones de Mensalão y operación Lava Jato y posteriores, ya que el desmantelamiento del fraude financiero y la corrupción comprobada y el lavado de dinero demuestran la necesidad de un nuevo entorno empresarial, que requiere transparencia e integridad en la conducción de los negocios. Los castigos y detenciones de altos ejecutivos y altos funcionarios del gobierno ocurrieron a la luz de la nueva ley anticorrupción instituida en el país (GONSALES, 2016).

Los datos investigados por PwC Brasil (2020, p. 4) muestran que el Riesgo de Cumplimiento con respecto al fraude y los delitos económicos más comunes ocurren con organizaciones tanto a nivel nacional como internacional. Según esta investigación, se encontró que en Brasil se encuentran entre los tres tipos más comunes de fraude: (1) soborno y corrupción, (2) fraude contable y (3) fraude cometido por el consumidor. Desde que se realizó la encuesta en 2020, se demostró que en los

RC: 103916

Disponible: https://www.nucleodoconhecimento.com.br/administracion-deempresas/beneficios-del-cumplimiento 
últimos dos años (entre 2018 y 2019), los fraudes más comunes que aparecieron en las primeras posiciones fueron: (a) robos de activos, (b) fraude en compras y (c) soborno y corrupción.

A su vez, según PwC (2020, p. 4) los datos comparativos del ranking de fraudes que aparecen en las primeras posiciones en todo el mundo fueron: (1) fraude cometido por consumidores, (2) ciberdelincuentes y (3) robo de activos. Desde que se realizó la encuesta en 2020, se demostró que en los últimos dos años (entre 2018 y 2019), los fraudes más comunes que aparecieron en las primeras posiciones fueron: (a) fraude cometido por el consumidor, (b) fraude contable (c) competencia desleal, (d) fraude de recursos humanos y (e) soborno y corrupción.

La Figura 5 muestra los datos en las perspectivas interna y externa. 
Figura 5 - Los tipos de delitos más comunes

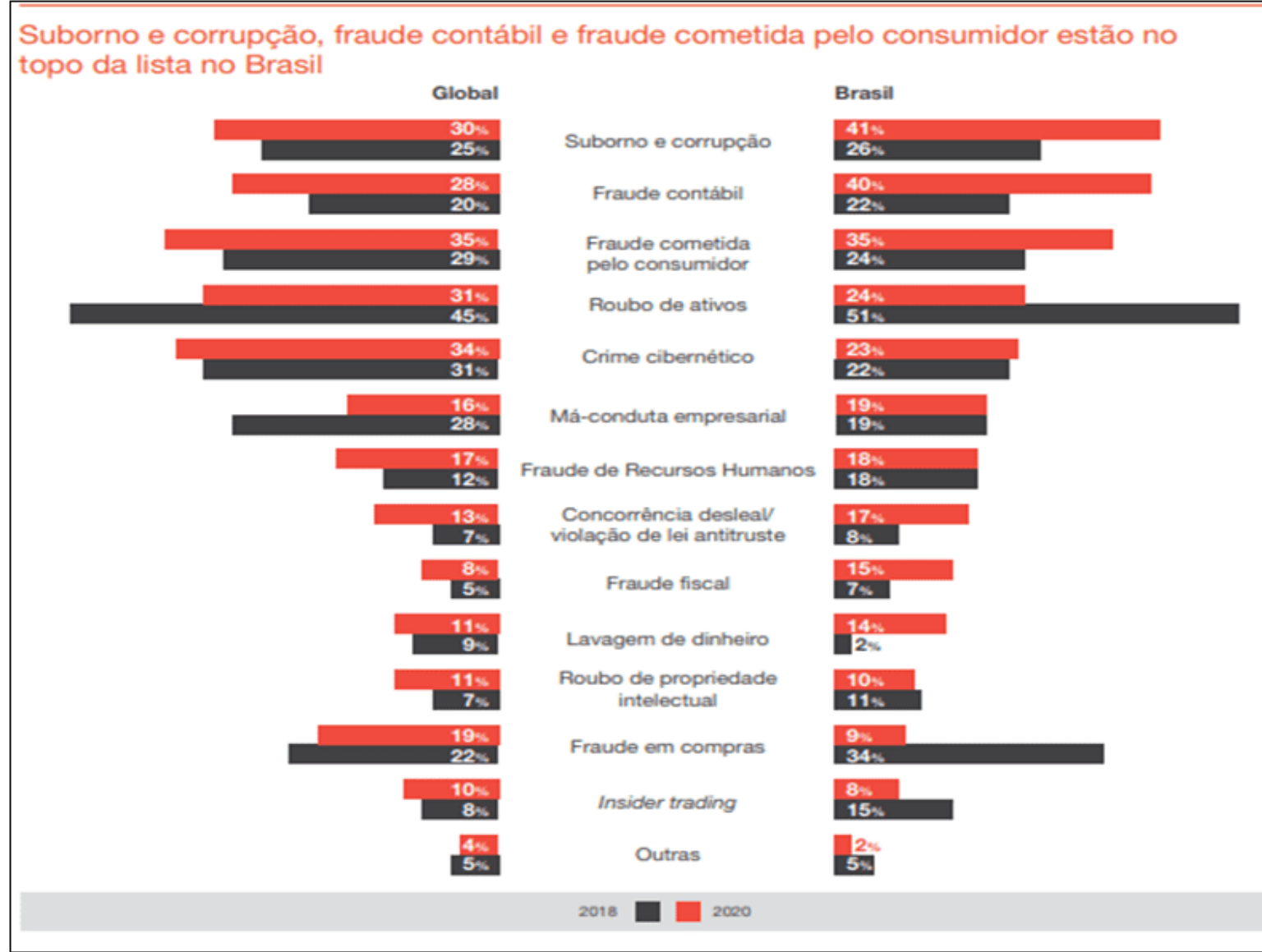

Fuente: PwC Brasil (2020, p. 5)

Son delitos económicos que, además de dañar el patrimonio de las organizaciones, comprometen su reputación e imagen, siendo posibles hasta llegar a la quiebra. Es en esta perspectiva que los programas de cumplimiento permiten evitar riesgos, conductas ilícitas y poco éticas, a partir de la implementación de un programa específico que contenga claramente el Código de Ética y Política Interna de la organización a seguir, confiriendo "crecimiento, inversión, credibilidad, seguridad y protección" (PwC, 2020).

En este sentido, Oliveira (2012 apud COSSENZO, 2015, p. 35) afirma que la atención de prevención debe estar compuesta básicamente por el compromiso que las empresas deben establecer internamente, relacionado con la conducta ética para las

RC: 103916

Disponible: https://www.nucleodoconhecimento.com.br/administracion-deempresas/beneficios-del-cumplimiento 
decisiones y comportamientos, impregnando sus procesos empresariales y operativos, e incluso pueden adoptar prácticas preventivas, relacionadas con la historia de los empleados, proveedores y clientes para, reforzar una cultura interna y externa de lucha contra el fraude.

Entre los riesgos del Cumplimiento, cabe mencionar la Teoría del Triángulo del Fraude, que destaca tres aspectos que potencian las posibilidades de actos ilícitos contra las organizaciones, son: presión, oportunidad y racionalización, según explican Condé, Almeida y Quintal (2015). Este triángulo se puede ver en la Figura 6.

Figura 6 - Triángulo de fraude

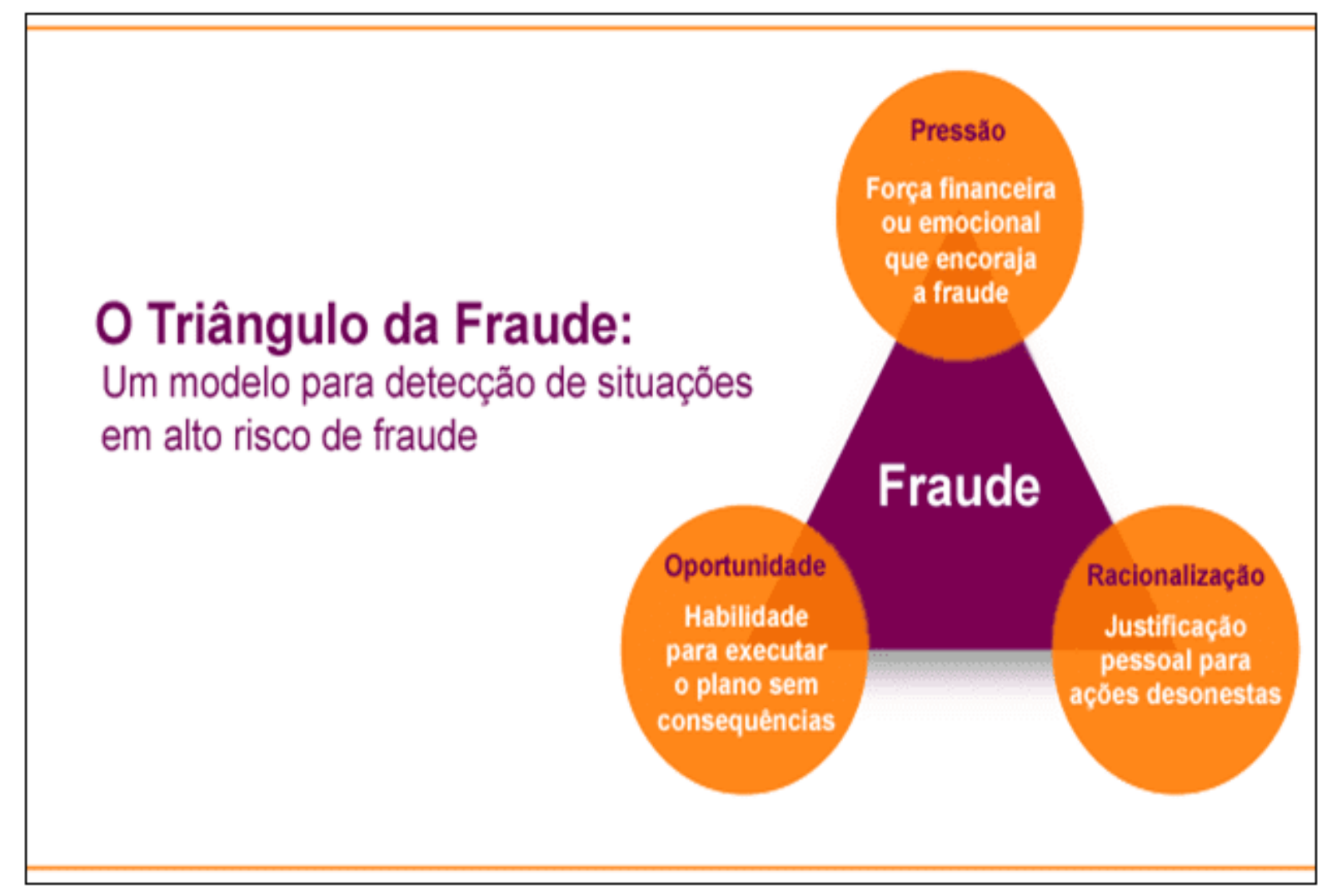

Fuente: Lustosa (2016)

Still Condé et al. (2015) definir cada uno de los elementos que componen el triángulo del fraude: 
a "pressão" geralmente está ligada ao motivo que leva ao crime, uma necessidade econômica "estressante" da empresa ou do funcionário. A "oportunidade" de cometer o ato ilícito, com baixo risco de ser descoberto ou nenhum. A "Racionalização" de uma autojustificação pelo cometimento do ilícito que para o fraudador seria "aceitável".

Ante este escenario, observamos la necesidad de un mapeo de riesgos organizacionales, a través de la prevención e identificación de riesgos, incluyendo el incumplimiento de la legislación y el código de conducta organizacional, riesgo operacional, riesgo de imagen por descuido o falta de conducta adecuada, o incluso riesgos externos como la demanda política del país, disrupciones tecnológicas, inestabilidad sanitaria y económica, aspectos que también pueden afectar a las organizaciones.

En este sentido, Cossenzo (2015) explica que para gestionar el fraude se necesitan estrategias que los identifiquen en el momento actual, además de otros, que permitan su prevención. Cuando se identifican los riesgos de fraude, también es necesario identificar cómo suceden y las personas que los practican, y para ello, es fundamental que las empresas compartan información entre sí.

Este mismo autor destaca la existencia de las Comisiones de Lucha contra el Fraude de la Federación Brasileña de Bancos (FEBRABAN), la Comisión base para la Inconsistencia de la Asociación Nacional de Instituciones de Crédito, Financiamiento e Inversión (ACREFI), y el acuerdo de cooperación firmado en 2009 entre la Policía Federal y Febraban, en lo que respecta a los controles electrónicos con información sobre los casos ocurridos (COSSENZO, 2015, p. 35).

Con respecto al fraude externo, Oliveira (2012, p. 73-4) referido por Cossenzo (2015, p. 36) enseña que existen tres fases preventivas: "prevención, detección y reacción", todas ellas en una fase integral, llamada "continua". Tales fases se pueden observar en el Gráfico 1. 
Cuadro 1 - Fases y categorías de la prevención del fraude externo

\begin{tabular}{|l|l|}
\hline Fase & Categorías relacionadas \\
\hline Continuo & Responsabilidad \\
\hline & Sinergia \\
\hline Mejora \\
\hline Prevención & $\begin{array}{l}\text { Institución } \\
\text { Identificación }\end{array}$ \\
\hline Detección & Mantenimiento \\
\hline Investigación \\
\hline Reacción & Sanciones \\
\hline
\end{tabular}

Fuente: Elaborado por el autor (2021)

Para prevenir tales ocurrencias, se deben adoptar acciones para sensibilizar a los involucrados, y se deben definir e implementar controles que inhiban nuevos incidentes; para su detección, deben adoptarse métodos de investigación y confirmación de sospechas sobre la ocurrencia o no de fraude; y para reaccionar, que las acciones sean establecidas por la empresa, cuando se encuentre fraude.

\subsection{SOLUCIONES ENTERPRISE GOVERNANCE, RISK MANAGEMENT AND COMPLIANCE (EGRC)}

Entre los beneficios que la implementación de un programa de Compliance y gestión de riesgos promueve dentro de las organizaciones se encuentran la protección contra el fraude e ilícito, promoviendo la mitigación de dichos riesgos.

Para que las medidas de seguridad sean efectivamente efectivas, a través de una realidad cada vez más tecnológica, es necesario que se desarrollen e implementen herramientas y soluciones para combatir el fraude, permitiendo un mayor cumplimiento de las prácticas necesarias para el éxito de las organizaciones, como explica Grand View Research (2014). Son medidas inherentes al Gobierno de las

RC: 103916

Disponible: https://www.nucleodoconhecimento.com.br/administracion-deempresas/beneficios-del-cumplimiento 
Tecnologías de la Información, así como al Internet of things (IoT), o internet de las cosas, que a su vez son inherentes al Gobierno Corporativo (CHAVES, 2014).

El Gobierno Corporativo consiste en un "sistema de relación entre accionistas, auditores independientes, ejecutivos de empresas y directores liderados por el consejo de administración" (LODI, 2000, referido por CHAVES, 2014, p. 21). A su vez, el Gobierno de TI, a través de "big data y analítica, loT, machine learning e IA y redes sociales, implica el intercambio de información personal, haciendo que los usuarios y las empresas sean más susceptibles a los hackers" (GRAND VIEW RESEARCH, 2014).

Los recursos aportados por ioT y digitalización de los procesos de negocio crecieron rápidamente, permitiendo interacciones de datos y proporcionando un mayor control de la información por parte de la dirección de las empresas. En la práctica, el objetivo es apoyarse en la Inteligencia Artificial (IA) para gestionar las obligaciones e informes externos, que permiten una mayor gestión de gobierno corporativo, riesgo y cumplimiento - EGRC (GRANDVIEW RESEARCH, 2014).

Según Chaves (2014, p.22), "para el Instituto Brasileño de Gobierno Corporativo (IBGC, 2006), el Gobierno de TI es un brazo del Gobierno Corporativo", que permite la elaboración e implementación de "regulaciones de alto impacto en el área de TI de las empresas, que requieren un mayor compromiso y eficacia en la planificación, controles, monitoreo y procesos de seguridad relacionados con las estrategias de negocio". Still Chaves (2014, p. 22) reporta una definición dada por el Information Technology Governance Institute, (ITGI, 2012) con respecto a los objetivos de LA Gobernanza de TI:

compreender os assuntos e a importância estratégica de TI, assegurar que a empresa possa sustentar suas operações e implementar as estratégias requeridas para expandir as suas atividades no futuro. As práticas de Governança de $\mathrm{TI}$ possibilitam que: sejam atendidas as expectativas dos clientes de $\mathrm{TI}$, que a performance de $\mathrm{TI}$ seja medida, os seus recursos possam ser gerenciáveis e os riscos do negócio mitigados.

RC: 103916

Disponible: https://www.nucleodoconhecimento.com.br/administracion-deempresas/beneficios-del-cumplimiento 
A su vez. Siqueira (2019) se refiere a la Encuesta de Ciberseguridad y Riesgo de Datos, elaborada por Marsh y McLennan en 2018, demostrando que solo el $18 \%$ de las pequeñas empresas habrían desarrollado algún tipo de plan de respuesta a incidentes cibernéticos.

En esta perspectiva, la creación e implementación de soluciones EGRC ofrecen la posibilidad de superar los desafíos existentes, como el fraude electrónico y la clonación, por ejemplo, en un universo empresarial "hiperconectado", que se puede obtener a través de software específico. También representa una herramienta que colabora sustancialmente en protección de datos y propiedad intelectual (PI), además de mejorar la gestión del trabajo remoto, un modelo híbrido tan común a lo largo de la pandemia por el Covid 19 (GRANDVIEW RESEARCH, 2014).

\section{BENEFICIOS DE UN PROGRAMA DE CUMPLIMIENTO Y GESTIÓN DE RIESGOS}

Según el portal 3Mind Jurídico (2021), entre los principales conceptos de cumplimiento y gestión deriesgos, se encuentra la generación de valor empresarial, que tiene como objetivo permitir la supervivencia y longevidad de las organizaciones. Entre los peligros e impactos financieros que la ausencia de un programa que promueva el buen gobierno se encuentran: "ausencia de lineamientos normativos; la falta de instrumentos de prevención adecuados; desalineaciones con las leyes aplicables; sistema de información sin operaciones estructuradas; gestión de procesos fallidos".

Dado que las instituciones públicas y privadas deben guiar su gestión en los programas de Gobierno Corporativo, Klen (2009, apud SENNO et al., 2019, p. 225) define que "el gobierno corporativo es el sistema por el cual las corporaciones comerciales son administradas y controladas".

En este sentido, todavía Senno et al. (2019) explican que las prácticas de gobierno corporativo deben converger para garantizar mecanismos que permitan el retorno

RC: 103916

Disponible: https://www.nucleodoconhecimento.com.br/administracion-deempresas/beneficios-del-cumplimiento 
financiero de las inversiones realizadas, mediante la adopción de normas jurídicas y la rendición de cuentas a todas las partes interesadas.

A su vez, Azevedo et al. (2017, p. 181) explicar que la gobernanza se refiere al gobierno, mientras que el gobierno corporativo se refiere al sistema por el cual los órganos y poderes se organizan dentro de una empresa", que debe estar respaldado por cuatro principios: transparencia, integridad o equidad, rendición de cuentas y respeto a las leyes.

Estas explicaciones tienen como objetivo colaborar para comprender mejor las prácticas de buen gobierno, que, según KPMG (2021), requieren de algunos elementos específicos para conformar un programa eficiente de Cumplimiento Normativo, que son:

1. Política y programa de cumplimiento;

2. Mensaje de liderazgo y cultura de cumplimiento;

3. Estructuración del oficial de cumplimiento;

4. Comunicación y capacitación de la política y el programa de cumplimiento;

5. Monitoreo, auditoría y evaluación de la efectividad de la política y programa de ética y cumplimiento;

6. Incentivos para el desempeño y acciones disciplinarias;

7. Investigación y prevención.

En esta perspectiva, se informa que un programa de cumplimiento implica estándares anticorrupción, antisoborno y de cumplimiento público. Para más detalles, se enumeran los beneficios proporcionados por Compliance, según el portal 3Mind Jurídico (2021):

1. Atraer inversores e inversiones;

2. Aumentar la gobernanza en la empresa;

3. Consolidar la cultura organizacional;

4. Corregir eficazmente las no conformidades;

5. Ganar credibilidad;

RC: 103916

Disponible: https://www.nucleodoconhecimento.com.br/administracion-deempresas/beneficios-del-cumplimiento 
6. Obtener ventaja competitiva sobre la competencia;

7. Identificar riesgos y prevenir problemas;

8. Mejorar la eficiencia y calidad de los productos y servicios ofrecidos;

9. Sostenibilidad.

A su vez, Caovilla (2017) describe algunos beneficios relacionados con la adopción del cumplimiento por parte de las organizaciones (nuestros grifos):

1. Nueva oportunidad de negocio y ventaja competitiva: las empresas que operan de acuerdo con las leyes y buenas prácticas buscan relacionarse con pares iguales, además de aumentar sus oportunidades en relación con aquellos que no son usuarios de programas de cumplimiento;

2. Nuevas inversiones: las organizaciones con bajas tasas de participación en escándalos ilícitos y sociales, que demuestran solidez, son más atractivas para los inversores internos y externos;

3. Mitigación de riesgos: las empresas que adoptan un programa de cumplimiento, además de identificar posibles riesgos para su negocio, son capaces de desarrollar e implementar acciones para mitigar posibles fallas y riesgos;

4. Corrección de no conformidades: además de las acciones preventivas para evitar posibles no conformidades, están las acciones para corregir las no conformidades identificadas en los procesos internos de una organización. Tales acciones necesarias para preservar la imagen y la reputación de una empresa pueden ser: corrección del plan de acción en curso; capacitación para empleados; revisión de políticas, procedimientos, conducta, empleados y proveedores y proveedores de servicios;

5. Cumplimiento as people awareness: una práctica que permite mitigar el fraude, ya que si los empleados y otros actores están capacitados en cumplimiento, son capaces de ver no conformidades dentro y fuera de la empresa, es decir, en competencia, en proveedores, prestadores de servicios;

6. El compliance como limitación de responsabilidad: los conocimientos adquiridos en formación y prácticas éticas relacionadas con el

RC: 103916

Disponible: https://www.nucleodoconhecimento.com.br/administracion-deempresas/beneficios-del-cumplimiento 
cumplimiento permiten la selección de mejores socios comerciales, además de proteger la reputación de una empresa. Dado que las buenas prácticas impregnan las rutinas comerciales de una organización, cualquier no conformidad puede conducir a la reducción de las sanciones que se le pueden aplicar;

7. Cumplimiento como sostenibilidad empresarial:la adopción y el mantenimiento de prácticas éticas y transparentes permiten a las organizaciones permanecer a largo plazo.

\section{CONCLUSIÓN}

A lo largo del análisis de las referencias teóricas seleccionadas para el desarrollo de este artículo, fue posible observar que un programa de cumplimiento y Gestión de Riesgos tiene alta importancia para la correcta gestión de procesos, prácticas y rutinas dentro de las organizaciones, involucrando a todos sus sectores y personal.

Volviendo al punto de las riendas; ¿Cómo colaboran los beneficios que ofrece el programa de cumplimiento y Gestión de Riesgos para reducir los riesgos de fraude, delitos ilícitos y corrupción dentro de las organizaciones e instituciones gubernamentales? Concluimos que, dado que la literatura demuestra que existen fraudes en todas las organizaciones, sin distinción por el segmento de actividad o su tamaño, el beneficio que el cumplimiento confiere a las empresas está precisamente en la protección que ofrece, al mitigar los diferentes tipos de riesgos e ilícitos, incluyendo evitar sanciones legales.

Con base en las leyes instituidas, a partir de la Ley Pionera de Lucha contra la Corrupción FCPA (Foreign Corrupt Practices Act), lospaíses signatarios de la OCDE, enumerados en este artículo, han adoptado iniciativas similares para promover la mitigación del fraude, la corrupción y las acciones poco éticas en sus gobiernos y organizaciones, fuertemente respaldados por las restricciones y castigos que traen las leyes.

RC: 103916

Disponible: https://www.nucleodoconhecimento.com.br/administracion-deempresas/beneficios-del-cumplimiento 
Además, surgió el desarrollo de nuevos instrumentos tecnológicos, apoyados por el Gobierno de las Tecnologías de la Información, para apoyar el Gobierno Corporativo, promoviendo cambios en la gestión de las organizaciones y sus procesos administrativos.

Dicha gestión es algo que debe basarse en herramientas modernas, no estando restringida a los procesos en sí, sino que debe permitir a una organización anticiparse a eventos futuros, evitando malentendidos.

En este contexto, las soluciones de EGRC, que permiten las mejores prácticas para el Gobierno de Tl y el Gobierno Corporativo, colaboran para la prevención y mitigación del fraude y las acciones no advertidas, protegiendo el crecimiento de las organizaciones y su imagen de mercado.

\section{REFERENCIAS}

AZEVEDO, Mateus Miranda de; CARDOSO, Antonio Almeida; DUARTE, Jairo Gonçalves; FEDERICO, Bianca Ellen; LIMA, Marco Antonio Ferreira. O Compliance e a gestão de riscos nos processos organizacionais. Revista de Pós-Graduação Multidisciplinar, São Paulo, v. 1, n. 1, p. 179-196, mar./jun. 2017.

ISSN 2594-4800 | e-ISSN 2594-4797 | doi: 10.22287/rpgm.v1i1.507; acesso em 08 dez 2021.

CAOVILLA, Renato Vieira. Benefícios práticos do compliance. 10 jul 2017. Disponível em: [https://www.migalhas.com.br/depeso/261662/beneficios-praticos-docompliance]; 08 dez 2017.

CHAVES, Elisabete Cecília Januário. Resultados e tendências de Requisitos e Práticas de Governança de TI nos bancos de Varejo no Brasil. Dissertação [Mestrado em Gestão e Tecnologia em Sistemas Produtivos] apresentada ao Centro Estadual de Educação Tecnológica Paula Souza, no Programa de Mestrado Profissional em Gestão e Tecnologia em Sistemas Produtivos. São Paulo, novembro

RC: 103916

Disponible: https://www.nucleodoconhecimento.com.br/administracion-deempresas/beneficios-del-cumplimiento 
de

2014.

Dsponível

em:

[http://www.pos.cps.sp.gov.br/files/dissertacoes/file/143/7fc218f434af24b99f50af42e 8442903.pdf]; acesso em 12 out 2021.

COELHO, Cláudio Carneiro Bezerra Pinto; SANTOS JÚNIOR, Milton de Castro. Compliance, FGV/IDE, 2021.

CONDÉ, Robson Augusto Dainez; ALMEIDA, Carlos Otávio Ferreira de; QUINTAL, Renato Santiago. Fraude Contábil: análise empírica à luz dos pressupostos teóricos do triângulo da fraude e dos escândalos corporativos. Gestão \& Regionalidade - Vol. 31 - № 93 - set-dez/2015. doi: 10.13037/gr. vol31n93.2929; acesso em 28 out 2021

COSSENZO, Fábio. FRAUDE BANCÁRIA: A mitigação dos Riscos e Perdas Financeiras no Segmento de Crédito Consignado. Monografia de Pós-Graduação Lato Sensu - MBA Gestão empresarial, apresentada à Fundação Instituto de Administração - FIA. São Paulo, 2015.

DURÃES, Cintya Nishimura; RIBEIRO, Maria de Fátima. O Compliance no Brasil e a Responsabilidade Empresarial no combate à corrupção. Revista Direito em Debate: Revista do Departamento de Ciências Jurídicas e Sociais da Unijuí Editora Unijuí - Ano XXIX - n. 53 - jan./jun. 2020. Disponível em: [http://dx.doi.org/10.21527/2176-6622.2020.53.69-78]; acesso em 01 dez 2021.

ENTERPRISE GOVERNANCE, RISK \& COMPLIANCE MARKET SIZE. Share \& Trends Analysis Report By Component, By Software, By Services, By Enterprise Type, By Vertical, And Segment Forecasts, 2021 - 2028

FGV - FUNDAÇÃO GETÚLIO VARGAS. Surgimento do Compliance. 2021.

GONSALES, Alessandra. O que é compliance? Publicação da Legal, Ethics and Compliance (LEC). Vídeo publicado em 29 fev 2016. Disponível em: [https://www.youtube.com/watch?v=2BDpJ6UMXb4]; acesso em 01 dez 2021.

RC: 103916

Disponible: https://www.nucleodoconhecimento.com.br/administracion-deempresas/beneficios-del-cumplimiento 
GRAND VIEW RESEARCH. Relatório de governança corporativa, risco e conformidade do mercado, análise de compartilhamento e tendências por componente, por software, por serviços, por tipo de empresa, por vertical e por segmento. Previsões, 2021-2028. Artigo publicado em abril de 2021. Disponível em: [https://www.grandviewresearch.com/industry-analysis/enterprise-governance-riskcompliance-egrc-market]; acesso em 20 nov 2021.

HAUSSEN, Leonard. Compliance. Reunião On-Line (ROL), Aula Organizada pela Fundação Getúlio Vargas, Escola de Administração de Empresas de São Paulo, São Paulo, Brasil. Agosto de 2021.

JORGE, Glaucio Fiorenzano; TOMAZ, Roberto Epifânio. Compliance rins - como implantar e quais os benefícios do programa de compliance? IV Congresso Catarinense de Direito processual Civil \& Mais. 7 a 9 de novembro de 2018. Univali Eventos - Universidade Vale do Itajaí. Disponível em: [https://core.ac.uk/download/pdf/276005736.pdf]; acesso em 20 out 2021.

KPMG - Compliance analytics. 2018. Disponível em: [https://assets.kpmg/content/dam/kpmg/br/pdf/2018/08/br-compliance-analytics.pdf]; acesso em 08 dez 2021.

KPMG - Compliance regulatório. 2021. Disponível em: [https://home.kpmg/br/pt/home/servicos/consultoria/risk-consulting/internal-auditrisk/compliance-regulatory.html]; acesso em 08 dez 2021.

MELO, Luísa. 8 crises empresariais causadas por funcionários brincalhões. Matéria publicada em 14 mar 2014. Disponível em: [https://exame.com/negocios/8crises-empresariais-causadas-por-funcionarios-brincalhoes/]; acesso em 20 out 2021.

MICHELOTTI, Andriws Loreto. 0 crime de lavagem de capitais e os desafios empresariais diante da implementação dos programas de criminal Compliance. Trabalho de Conclusão de Curso [Bacharelado em Direito] apresentado à Faculdade Antonio Meneghetti (AMF). Restinga Seca (RS), 2020. Disponível em:

RC: 103916

Disponible: https://www.nucleodoconhecimento.com.br/administracion-deempresas/beneficios-del-cumplimiento 
[http://repositorio.faculdadeam.edu.br/xmlui/bitstream/handle/123456789/673/TCC_D IR_ANDRIWS_MICHELOTTI_AMF_2020.pdf?sequence=1\&isAllowed $=y$ ]; acesso em 07 dez 2021.

NASCIMENTO, Juliana Oliveira. A Função Sociald da Empresa e a Efetividade Prática da Conduta de Integridade: o Compliance Vivo. Dissertação [mestrado em Direitos Fundamentais e Democracia] apresentada ao Centro Universitário Autônomo do Brasil. Curitiba, 2018. Disponível em: [https://www.unibrasil.com.br/wpcontent/uploads/2019/07/Dissertação-JULIANA-OLIVEIRA-NASCIMENTO.pdf]; acesso em 29 ago 2021.

NASCIMENTO, Débora Minuncio. Evolução histórica e legislações acerca do Compliance. Artigo publicado em 07 nov 2021. Disponível em: [https://advocaciadeboramn.jusbrasil.com.br/artigos/700763578/evolucao-historica-elegislacoes-acerca-do-compliance]; acesso em 20 out 2021.

\section{OECE - ORGANIZAÇÃO PARA A COOPERAÇÃO E DESENVOLVIMENTO ECONÔMICO INTERNACIONAL. \\ Disponível \\ em:}

[https://www.dgae.gov.pt/servicos/comercio-internacional-e-relacoesinternacionais/multilaterais/organizacao-para-a-cooperacao-e-desenvolvimentoeconomico-ocde-.aspx]; acesso em 08 dez 2021.

PwC. Combate a fraudes e crimes econômicos: uma batalha sem fim Pesquisa Global sobre Fraudes e Crimes Econômicos 2020. Disponível em: [https://www.pwc.com.br/pt/publicacoes/servicos/assets/consultorianegocios/2020/pesquisa_sobre-fraudes-e-crimes-economicos-2020-pwc-brasil.pdf]; acesso em 20 out 2021.

SENNO, Elisângela Pereira; PRATES, Glaucia Aparecida; LUCENTE, Adriano dos Reis; GALLI, Lesley Carina do Lago Attadia; GALLI, Rafael Altafin. Benefícios da implantação do programa de Compliance em uma organização do setor de energia: um estudo de caso. II SITEFA - Simpósio de Tecnologia Fatec Sert5aozinho. Disponível em:

RC: 103916

Disponible: https://www.nucleodoconhecimento.com.br/administracion-deempresas/beneficios-del-cumplimiento 
[https://sitefa.fatecsertaozinho.edu.br/index.php/sitefa/article/view/90/51]; acesso em 08 dez 2021.

SIQUEIRA, Marina Paiva de. Cyber Risks: Fatores decisórios para a transferência mediante a contratação do seguro. Monografia [Bacharelado em Administração] apresentada ao Departamento de Administração da Universidade Federal Fluminense. Niterói, RJ. 2019.

SOBREIRA FILHO, Enoque Feitosa; LEITE, Flavia Piva Almeida; MARTINS, José Alberto Monteiro. Ética empresarial como base de sustentação do programa de Compliance: uma breve análise sobre a ética, a integridade e o Compliance. Revista Relações Internacionais no Mundo Atual. Vol. 2,n. 23, 2019. Disponível em: [http://revista.unicuritiba.edu.br/index.php/RIMA/article/view/3891/371373033]; acesso em 29 ago 2021.

SOUZA, Silvia Regina; MACIEL-LIMA, Sandra; LUPI, André Lipp Pinto Basto. Aplicabilidade do Compliance na Administração Pública em face ao momento político atual brasileiro. Percurso - Anais do I Congresso Ibro-Americano de Direito Empresarial e Cidadania (CONIBADEC), vol. 01, n. 24, Curitiba, p.1-22, 2018. Disponível em:

[http://revista.unicuritiba.edu.br/index.php/percurso/article/view/3274/371371782]; acesso em 01 dez 2021.

3MIND JURÍDICO. Compliance: o que é e o benefício para empresas. Abril de 2021. Disponível em: [https://www.3mind.com.br/blog/compliance-empresas/]; acesso em 08 dez 2021.

VAZ, Tatiana. 5 empresas envolvidas em escândalos recentes. Artigo publicado em 30 abr 2012. Disponível em: [https://exame.com/negocios/5-empresas-envolvidasem-escandalos-recentes/]; acesso em 28 out 2021.

RC: 103916

Disponible: https://www.nucleodoconhecimento.com.br/administracion-deempresas/beneficios-del-cumplimiento 
VEJA ON LINE. Funcionário de restaurante publica foto lambendo comida de clientes. Junho de 2013.2 Disponível em: [https://veja.abril.com.br/economia/funcionario-de-restaurante-publica-fotolambendo-comida-de-clientes/]; acesso em 14 out 2021.

Enviado: Noviembre de 2021.

Aprobado: Diciembre de 2021. 